\title{
Acute Spinal Cord Compression due to Chloroma as the Initial Presenting Feature of Acute Myeloid Leukemia
}

\author{
Sushant Kumar Patro ${ }^{1}$ Biswaranjan Nayak ${ }^{2}$ Arun Kumar ${ }^{3}$ \\ Prafulla Kumar Sahoo ${ }^{5}$ \\ ${ }^{1}$ Department of Neurosurgery, Ispat General Hospital, Rourkela, \\ Odisha, India \\ 2Department of Neurosurgery, AMRI Hospital, Bhubaneswar, Odisha \\ ${ }^{3}$ Department of Neurosurgery, The Neurocity Hospital, Varanasi, \\ Uttar Pradesh \\ ${ }^{4}$ Department of Neurosurgery, Max Hospital, Patparganj, Delhi \\ ${ }^{5}$ Department of Neurosurgery, Apollo Hospital, Bhubaneswar, \\ Odisha \\ Indian J Neurosurg 2019;8:130-132
}

Balappa Holeppagol Krishnamurthy ${ }^{4}$ Debabrata Biswal ${ }^{5}$

\begin{abstract}
Address for correspondence Biswaranjan Nayak, D Orth, MS, DNB (Neurosurgery), Associate Consultant, Department of Neurosurgery, AMRI Hospital, Bhubaneswar 751030, Odisha, India (e-mail: dr.bnayak@gmail.com).
\end{abstract}

\begin{abstract}
Keywords

- chloroma

- leukemia

- paraplegia

Chloroma is the deposits in leukemic cells outside the bone marrow and is not common. It is extremely rare to find a chloroma compressing the spinal cord causing paraplegia without any prior symptoms. Only few case reports have been found in the literature till date. We report an interesting case of a 7-year-old boy who presented with acute back pain and paraplegia with a dorsal extradural mass, and with a previous diagnosis of tuberculosis of the dorsal spine, treated with antitubercular drugs without any other signs of systemic illness. After surgical decompression and histopathologic examination of the tissue, it was found to be chloroma. There are only few reported cases of this disease initially presented as paraplegia without any systemic signs of malignancy in the literature, and chloroma presents as a diagnostic challenge to the surgeon. Chloromas are a rare cause of acute spinal cord compression but should be diagnosed and treated promptly to prevent neurologic dysfunction.
\end{abstract}

\section{Introduction}

Chloroma is the deposit of leukemic cells outside the bone marrow. It can arise anywhere but usually occurs in the bone, skin, and lymph nodes. ${ }^{1}$ Occurrence in the central nervous system (CNS) and spinal cord is uncommon. ${ }^{2}$ Once the incident occurs, neurologic dysfunction progressively becomes worse. Hence, emergency surgical decompression is advised as a treatment to make neural function recover. Biopsy of the lesion helps in establishing the diagnosis and appropriate management. These lesions are usually misdiagnosed and treated as other granulomatous lesions, for example tuberculosis (TB). Herein, we report a case of a patient with chloroma with acute extradural spinal cord compression.

\section{Case Report}

A 7-year-old boy arrived in our emergency department with acute onset of paraplegia. He had a history of back pain for 30 days and weakness of both lower limbs along with frequency in micturition and constipation since 15 days, and significant weight loss. He consulted local physician, for which he was advised magnetic resonance imaging (MRI) of the spine, which revealed a lesion of $60 \times 7 \mathrm{~mm}$ in the epidural space from D4-D7 with cord compression (- Fig. 1a) and gave an impression of TB of the spine with cords compression. Then, he was given antitubercular therapy. Despite the treatment, his neurologic status worsened rapidly so he was brought to us in a bed-ridden condition. On examination he had sensory deficit below D6, spastic paraparesis both lower received

October 27, 2018

accepted

December 4, 2018

published online

August 27, 2019
DOI https://doi.org/ 10.1055/s-0039-1695086 ISSN 2277-954X.
(C)2019 Neurological Surgeons'

Society of India
License terms

() (1) $\Theta \circledast$ 


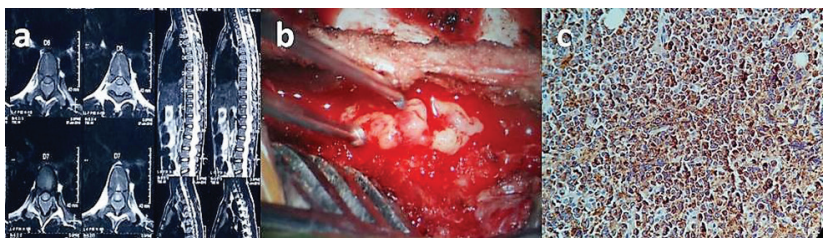

Fig. 1 (a) MRI of spine revealed a lesion of $60 \times 7 \mathrm{~mm}$ in the epidural space from D4-D7 with cord compression. (b) We have done hemilaminectomy of D4-6 and after excising the ligamentum flavum, a thick, cheesy, yellowish, semisolid, organized material. (c) Myeloperoxidase positivity.

limbs with power $3 / 5$, with bowel and bladder involvement. After routine hematologic examination and coagulation profile, which were essentially normal, we planned for an emergency decompression of the spinal cord by laminectomy with cord decompression and biopsy of the lesion bearing a pathologic diagnosis of spine TB in mind. We had done hemilaminectomy of D4-6, and after excising the ligamentum flavum, a thick cheesy, yellowish, semisolid, organized material protruded out ( $\mathbf{- F i g}$. 1b). It was adherent to the dura and was completely removed. The tissue was sent for microbiologic and histopathologic examination. In the postoperative period, the patient recovered well and was discharged on third postoperative day. We reevaluated the case on 10th postoperative day. He improved in strength and walked with support. The wound was healthy. The Gram stain, acid-fast bacilli (AFB) stain, and TB polymerase chain reaction (PCR) were negative. The pathologist gave the report as non-Hodgkin's lymphoma due to the presence of large round to oval tumor cells with pleomorphic convoluted vesicular nuclei with brisk mitosis and frequent apoptotic bodies. Therefore, we decided to take the help of the oncologist, who advised ultrasonography of the abdomen and peripheral smear. Ultrasonography revealed no organomegaly, but there were blast cells $(11 \%)$ in the peripheral smear. Hence bone marrow aspirate study was done. The bone marrow was hypercellular, erythropoiesis has reduced, and the marrow was replaced with blast cells (90\%). The cells are large with the nucleus having irregular nuclear margin, prominent nucleoli, and cytoplasmic granules. The above features are suggestive of acute myeloid leukemia (AML). Then the histopathologist reviewed the case and did immunohistochemistry. It became positive for CD38, CD64, CD33, HLA DR, CD13, CD117, and myeloperoxidase (MPO) (-Fig. 1c). The flow cytometry analysis also showed gated cells expressing CD34, CD38, CD64, CD33, HLA DR, CD117, and MPO. The final diagnosis was that the tissue is chloroma in a case of AML with CD38 positivity. The patient then started chemotherapy (daunorubicin, cytarabine) and completed the course of three cycles. We had not given radiotherapy as the patient showed AML in immunohistochemical examination. Now the patient is surviving even after 8 months of follow-up.

\section{Discussion}

Chloroma is the deposit of leukemic cells outside the bone marrow. They are generally seen in the ribs, sternum, pelvis, and orbital bones as well as in the soft tissues, lymph nodes, skin, and gums. ${ }^{2}$ Involvement of the CNS is rare, and spinal cord compression by chloromas is even rarer., ${ }^{2,3}$ Other names given to chloroma include granulocytic sarcoma, myeloid sarcoma, myelosarcoma, myeloblastoma, myelocytoma, chloroleukemia, and extramedullary myeloblastictumor. ${ }^{2}$ Dock made the first correlation between granulocytic sarcoma and leukemia in $1893 .^{3}$ In 1937, Kandel reviewed the literature and noted that chloromas were associated with myelogenousleukemia. ${ }^{4}$ Chloroma occurs in approximately $3 \%$ of AML patients, and rarely, it may be the presenting manifestation of undiagnosed leukemia or may even precede the development of leukemia in the bone marrow by months to years. ${ }^{5}$ Malignant spinal cord compression is an uncommon event occurring in $0.2 \%$ of all cancer patients at diagnosis with a cumulative incidence of $2.5 \%$ during the course of the disease. ${ }^{6} \mathrm{MRI}$ is the best choice for neuroimaging examination to show epidural tumor lesions. It is also the best neuroimaging examination to show the epidural tumor lesions without being specific. Granulocytic sarcoma is isointense in T1 and T2 with in general a contrast enhancement. Macroscopically, chloromas are green (hence the name) in appearance due to the MPO in the leukemia cells and fades when exposed to air. ${ }^{7-11}$ The correct diagnosis of chloroma is obtained in only approximately $50 \%$ of nonleukemic patients due to its rareness and histologic and radiologic similarities to malignant lymphoma. ${ }^{9}$ Clinically and histologically, chloromas should be differentiated from lymphomas. ${ }^{10}$ In immunohistochemical studies, especially staining of MPO, CDs are extremely helpful to make a correct diagnosis. Chloromas are usually radiosensitive and often treated with chemoradiotherapy. However, when presented with paraplegia as a presenting complain, immediate decompression of the spinal cord is the preferred mode of surgical therapy and should be warranted without wasting time. ${ }^{12,13}$ Early administration of anticancer therapy is beneficial.

\section{Conclusion}

Chloromas are rare lesions, more so in the extradural region of the spine. They may present as acute onset of paraplegia without any other major symptoms. It is usually confused with TB and other granulomatous lesions radiologically and with lymphomas histologically. Therefore after resection, proper immunohistochemistry is required for the exact diagnosis. Chemotherapy is the usual treatment for these lesions; however, some advise spinal axial radiotherapy.

\section{Note}

No financial support of any kind from anybody for this study was received.

\section{Contribution of the Authors}

Dr. Nayak: Concept, design, definition of intellectual content, literature search, clinical study, manuscript preparation, manuscript editing, manuscript review, guarantor, and corresponded with the editorial team; Dr. Patro, Dr. Kumar, Dr. Krishnamurthy, Dr. Biswal, and Dr. Sahoo: Literature search, clinical study, manuscript editing, manuscript review. 


\section{Declaration of Authors}

It is declared that the manuscript has been read and approved by all the authors, that the requirements for authorship as stated earlier in this document have been met, and that each author believes that the manuscript represents honest work.

\section{Conflicts of Interest}

There is no conflict of interest.

\section{References}

1 Brunning RD, Matutes E, Fladrin G, et al. Acute myeloid leukemia not otherwise categorised. In: Jaffe ES, Harris NL, Stein H, Vardiman JW, eds. Pathology and Genetics of Tumors of Haematopoietic and Lymphoid Tissues. Lyon, France: IARC Press; 2001. World Health Organization Classification of Tumours, 3, pp 91-105

2 Shiozawa Y, Kiyokawa N, Saito M, Fujimoto J, Hata J, Yamashiro Y. Granulocytic sarcoma of the spine in a child without bone marrow involvement: a case report and literature review. Eur J Pediatr 2005;164(10):616-620

3 Dock G. Chloroma and its relation to leukemia. Am. J Med Sci 1893;106:152-185

4 Kandel EV. Chloromas: review of the literature from 19261936 and report of 3 cases. Arch Intern Med 1937;59:691
5 Landis DM, Aboulafia DM. Granulocytic sarcoma: an unusual complication of aleukemic myeloid leukemia causing spinal cord compression. A case report and literature review. Leuk Lymphoma 2003;44(10):1753-1760

6 Loblaw DA, Laperriere NJ, Mackillop WJ. A population-based study of malignant spinal cord compression in Ontario. Clin Oncol (R Coll Radiol) 2003;15(4):211-217

7 Serefhanoglu S, Goker H, Aksu S, et al. Spinal myeloid sarcoma in two non-leukemic patients. Intern Med 2010;49(22):2493-2497

8 Amalraj P, Syamlal S. Unusual case of paraplegia. Ann Indian Acad Neurol 2009;12(3):188-190

9 Cui Y, Zhou JL, Wu JH, Zhang JZ. Synchronous granulocytic sarcoma of the breast and spine: a case report and review of the literature. Chin Med J (Engl) 2008;121(18):1854-1856

10 Ghalaut PS, Jindal S, Jeewan O. Granulocytic sarcoma as initial presentation of acute myeloid leukaemia. J Assoc Physicians India 2005;53:828-830

11 Chevallier P, Mohty MB, Lioure B, et al. Allogeneic hematopoietic stem cell transplantation for myeloid sarcoma: a retrospective study from the SFGM-TC. J Clin Oncol 2008;26:1-4

12 Chouchary DR, Bhattacharya M, Mishtra P, Mahapatra M, Kumar R. Granulocytic sarcoma of spine: an unusual initial presentation of acute myeloid leukemia. J Assoc Physicians India 2006;54:897-899

13 Buckland ME, Scolyer RA, Donellan MB, Brew S, McGee-Collett $M$, Harper CG. Spinal chloroma presenting with triplegia in an aleukaemic patient. Pathology 2001;33(3):386-389 